ARTICLE OPEN

\title{
Exploring salivary diagnostics in COVID-19: a scoping review and research suggestions
}

\author{
Priyanka Kapoor ${ }^{1}$, Aman Chowdhry $\mathbb{D}^{2}$, Om Prakash Kharbanda ${ }^{3}$, Deepika Bablani Popli $\mathbb{D}^{2}$, Kamini Gautam ${ }^{4}$ and Vikram Saini (D) $^{4}$
}

\begin{abstract}
INTRODUCTION: Molecular diagnostics for SARS-CoV-2 infection characteristically involves the sampling of the throat or nasopharyngeal swab (NPS). However, these procedures are invasive, require necessary skills for sample collection, cause patient discomfort, and are non-conducive for extensive scale testing. Saliva is increasingly being suggested as an alternate diagnostic sample in SARS-CoV-2 infection.

OBJECTIVES: This scoping review was done with the objective of exploring the evidence on the role of saliva as an alternate diagnostic sample in SARS-CoV-2 condition.

METHODS: Thorough search of the literature in major databases was undertaken in June 2020 using free text and MESH terms, followed by PRISMA to identify 17 studies for data extraction.

RESULTS AND CONCLUSIONS: Evidence was summarised for study characteristics, salivary sampling characteristics, viral load, and longevity of virus in saliva. The literature supports that saliva offers a simple sample collection method compared to techniquesensitive NPS and has the advantage of point-of-care testing for initial screening in community or hospital-based set-up. The additional highlights of this review are heterogeneity in the current literature and the gaps in methodology. Therefore, a robust study design to generate higher levels of evidence has been proposed.
\end{abstract}

BDJ Open (2021)7:8

; https://doi.org/10.1038/s41405-021-00064-7

\section{INTRODUCTION}

Coronaviruses (CoVs) belong to a group of zoonotic RNA beta-CoV that primarily circulate among animals but can infect humans too. ${ }^{1,2}$ $\mathrm{CoV}$ as described on cryogenic electron microscopic images bear crown-like spikes on its surface ${ }^{3}$ and have been classified into four groups, namely, alpha, beta, gamma, and delta CoV. The alpha and beta variety of CoV infects mainly the human's and mammal's respiratory, gastrointestinal, and central nervous system. Gamma and delta types of CoV infects mostly birds. ${ }^{4,5}$

Till December 2019, six CoVs were known to infect humans out of which two were a variety of alpha CoV [229E, NL63] and four were beta CoV [OC43, HKU1, Middle East respiratory syndrome (MERS), severe acute respiratory syndrome (SARS)]. ${ }^{2}$ Additional strain of CoV (seventh) affecting humans emerged in Wuhan (Hubei province), China from the Huanan seafood wholesale market on December 8, 2019., 3 This strain was named as "severe acute respiratory syndrome coronavirus 2" (SARS-CoV-2) or coronavirus disease 2019 (COVID-19) by International Committee on Taxonomy of Viruses and World Health Organisation (WHO), who proclaimed it as a pandemic on March 11, 2020.

The molecular structure of virion of SARS-CoV-2 has a diameter $\sim 50-200 \mathrm{~nm}^{3}$ comprises of four structural proteins: (1) Spike (S); (2) Envelope (E); (3) Membrane (M); and (4) the nucleocapsid $(\mathrm{N})$. Of these, $\mathrm{S}$ protein has a high affinity for human angiotensin-converting enzyme 2 (hACE2) receptors in the host cells and aids in its subsequent entry into the human body. ${ }^{8}$ The interactions between the host ACE2 receptors and SARS-CoV spike protein have been implicated for human to human transmission of the virus, ${ }^{9}$ but a comprehensive mechanism of its binding to SARS-CoV-2, leading to pathological damage, requires further investigation.

Viral diagnosis has progressed tremendously with a multitude of recent and more accurate techniques ranging from laboratory testing to advanced point of care. Of the various modalities for the SARS-CoV-2 diagnosis, the most reliable test is reverse transcription polymerase chain reaction (RT-PCR) on nasopharyngeal swabs (NPSs). ${ }^{10,11}$ However, NPS necessitates the availability of skilled technical staff, causes significant patient discomfort during test sample collection, and is associated with a high risk of infection to healthcare workers (HCWs) involved. ${ }^{12,13}$ Additional factors like psychological fear of collection and inadequate sampling technique may diminish specimen quality and lower test sensitivity.

Saliva is suggested to cause human-to-human transmission via droplet infection and may be used as an alternative to NPS for SARS-CoV-2 detection. Sabino-Silva et al. have compiled and proposed three different pathways for COVID-19 for reaching the oral cavity (Fig. 1): first, from upper and lower respiratory tract as a source; second, from blood having access to the mouth via crevicular fluid; third, from infected major and minor salivary glands. $^{14}$

Saliva may serve as a reliable alternative to NPS by offering advantages of self-collection of sample and countering issues like scarcity of NPS swabs and protective gear for concerned medical staff. ${ }^{14,15}$ Interestingly, some studies also suggest a positive result for SARS-CoV-2 in salivary specimens while NPS remains negative in paired samples. ${ }^{16,17}$

\footnotetext{
${ }^{1}$ Orthodontics, Faculty of Dentistry, Jamia Millia Islamia, New Delhi, India; ${ }^{2}$ Oral Pathology \& Microbiology, Faculty of Dentistry, Jamia Millia Islamia, New Delhi, India; ${ }^{3}$ Dr. C.G.

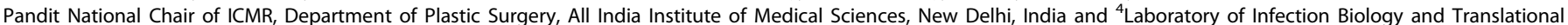
Research, Department of Biotechnology, All India Institute of Medical Sciences, New Delhi, India Correspondence: Deepika Bablani Popli (dpopli@jmi.ac.in)
} 


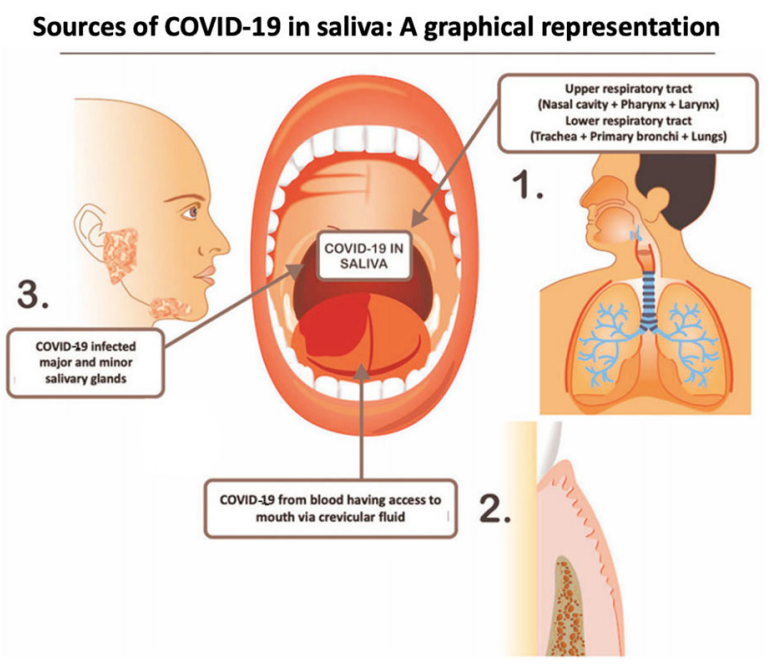

Fig. 1 Sources of COVID-19 in saliva. A graphical representation showing three significant pathways for COVID-19 to reach saliva. (1) from the secretions of the upper and lower respiratory tract, (2) from the blood via gingival crevicular fluid and, (3) from the secretions of the infected major and minor salivary glands.

Hence, the current scoping review was planned to analyse the feasibility of saliva as a diagnostic sample for detecting SARS-CoV2 infection. The objectives were to critically evaluate the current evidence related to salivary diagnostics in the progression of SARS-CoV-2 from an early stage of infection to recovery. The review also considers the strengths and shortcomings of salivary studies to deduce a robust study design to generate a higher level of evidence.

Search strategy and selection criteria

A scoping review of the literature was conducted to study the effective value of saliva samples for COVID-19 diagnosis. A thorough search of the literature was conducted in three databases Pubmed (P), Web of Science (WOS), and Scopus along with specialised COVID issues of PubMed (https://www.ncbi.nlm. nih.gov/sars-cov-2/), WHO (https://www.coronavirus.gov.https:// www.coronavirus.gov), and International Association of Dental Research COVID Resource in June 2020, along with hand search (HS) and reference tracking. The terms used for the search were MESH terms "Saliva", "Diagnosis", and free text terms "COVID", "SARS", and "Corona". The initial search revealed 43 articles in (P), 278 in WOS, 268 in Scopus along with 5 in (HS) and 2 in related search. The preferred reporting system of systematic reviews and meta-analysis (Fig. 2) criteria left us with 17 studies for data extraction. Using PICO (participants, intervention, comparator and outcome) criteria (Table 1), three researchers did data extraction individually, and the fourth researcher addressed any discordance.

\section{RESULTS}

This scoping review has broadly studied the presence of viral load in the saliva and its sensitivity in comparison to swab-based diagnostics. The results were used to establish the utility of a new sampling strategy, i.e. saliva-based molecular diagnostics in SARSCoV-2.

Study characteristics

The studies included in the review were mostly unicentric; a majority of them conducted in China. The sample size of 14 studies was $<100$, with 4 of them being case series/reports of 1 or 2 patients, ${ }^{18-21} 1$ study evaluated saliva in a single subject, ${ }^{22}$ and 3 studies had sample nearing or above $100 .^{23-25}$ RT-PCR was the

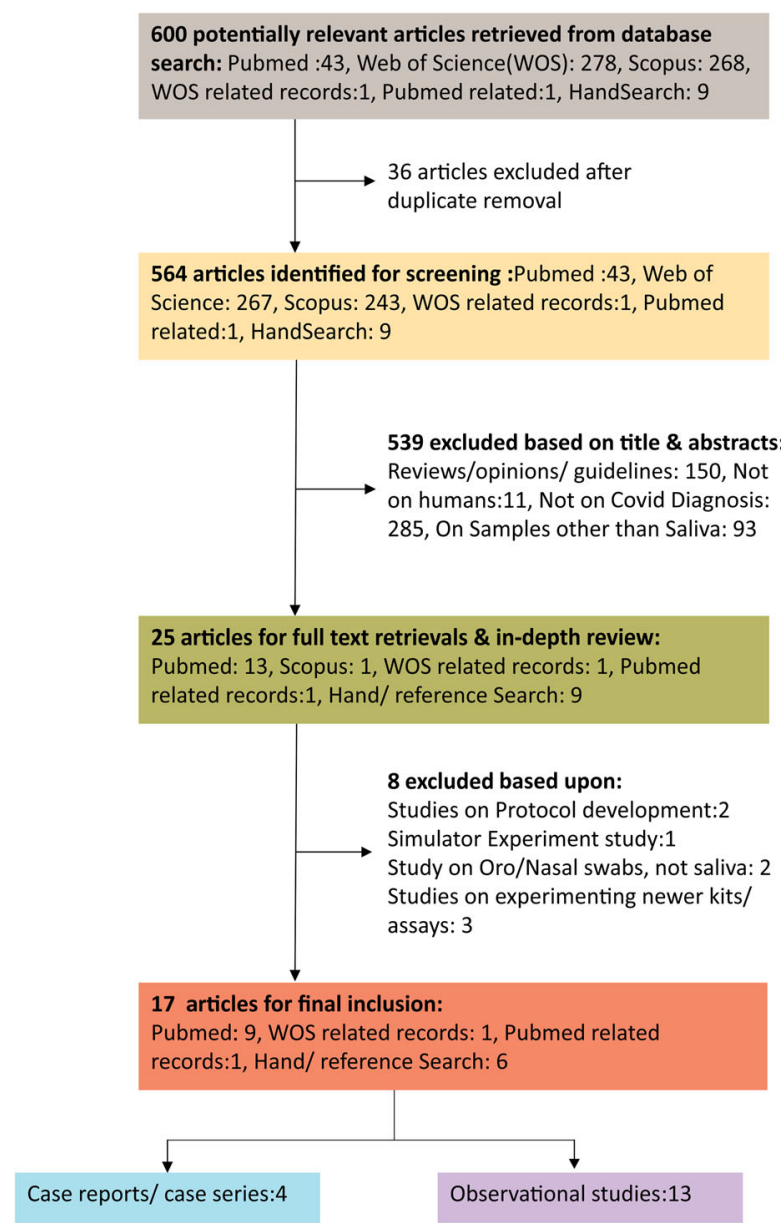

Fig. 2 Search strategy. Preferred reporting Items for systematic reviews and meta-analysis (PRISMA) was employed to search the literature from different sources.

most common technique utilised for identification of SARS-CoV-2. Twelve studies did not have control groups. Controls were used in 5 studies $\left[(n=12){ }^{26}(n=33){ }^{27}(n=50){ }^{28}(n=9\right.$ paired samples), ${ }^{16}$ pre-pandemic salivary samples $(n=134){ }^{29}$

Both cross-sectional and longitudinal observational cohorts have been used in the current scoping review to assess SARS-CoV-2 viral load in salivary samples at different stages or days from disease onset. While few studies did serial salivary sample collection in the same patient at different observation points, ${ }^{16-19,21,23,27,30}$ one study evaluated salivary viral load in different patients at different days of symptom onset. ${ }^{26}$

Viral load characteristics in serial sampling

Serial salivary specimens of 23 patients of severe and mild SARSCoV-2 presented with a median of $5.2 \log _{10}$ copies $/ \mathrm{mL}$ viral load and showed the maximum load during the initial 1 week of hospitalisation, only to decline after 1 week. $^{30}$ Another study, where serial samples were taken after a gap of 4 days, in 8 out of 25 severe and very severe disease patients showed $\mathrm{Ct}$ values (mean $27.16 \pm 3.07$ ) below a threshold value of 33 , both in first salivary swab and the repeat sample. ${ }^{17}$ High $\log _{10}$ count was also observed in saliva in 2 patients $\left(6.63 \log _{10}\right.$ copies $/ \mathrm{mL}$ and 7.10 $\log _{10}$ copies/mL in patients 1 and 2, respectively) who underwent repeat sampling every 2 days from hospital day 1 to $9 .^{19}$

Interestingly, in one study, samples were collected crosssectionally from different patients at different days of disease rather than repeat sample of the same patients. The study showed similar results with the highest count of $6.38 \times 10^{8}$ copies $/ \mathrm{mL}$ in 


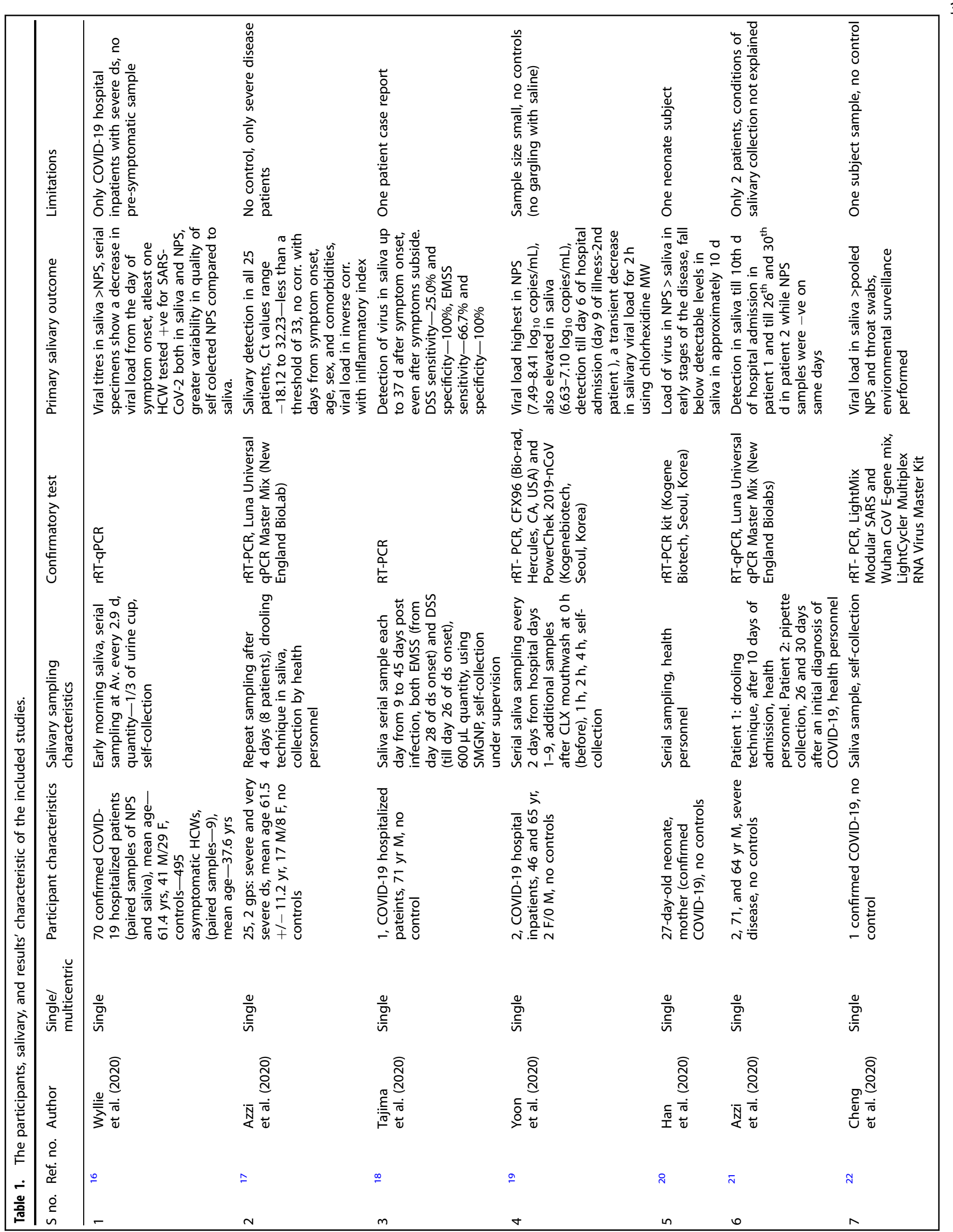




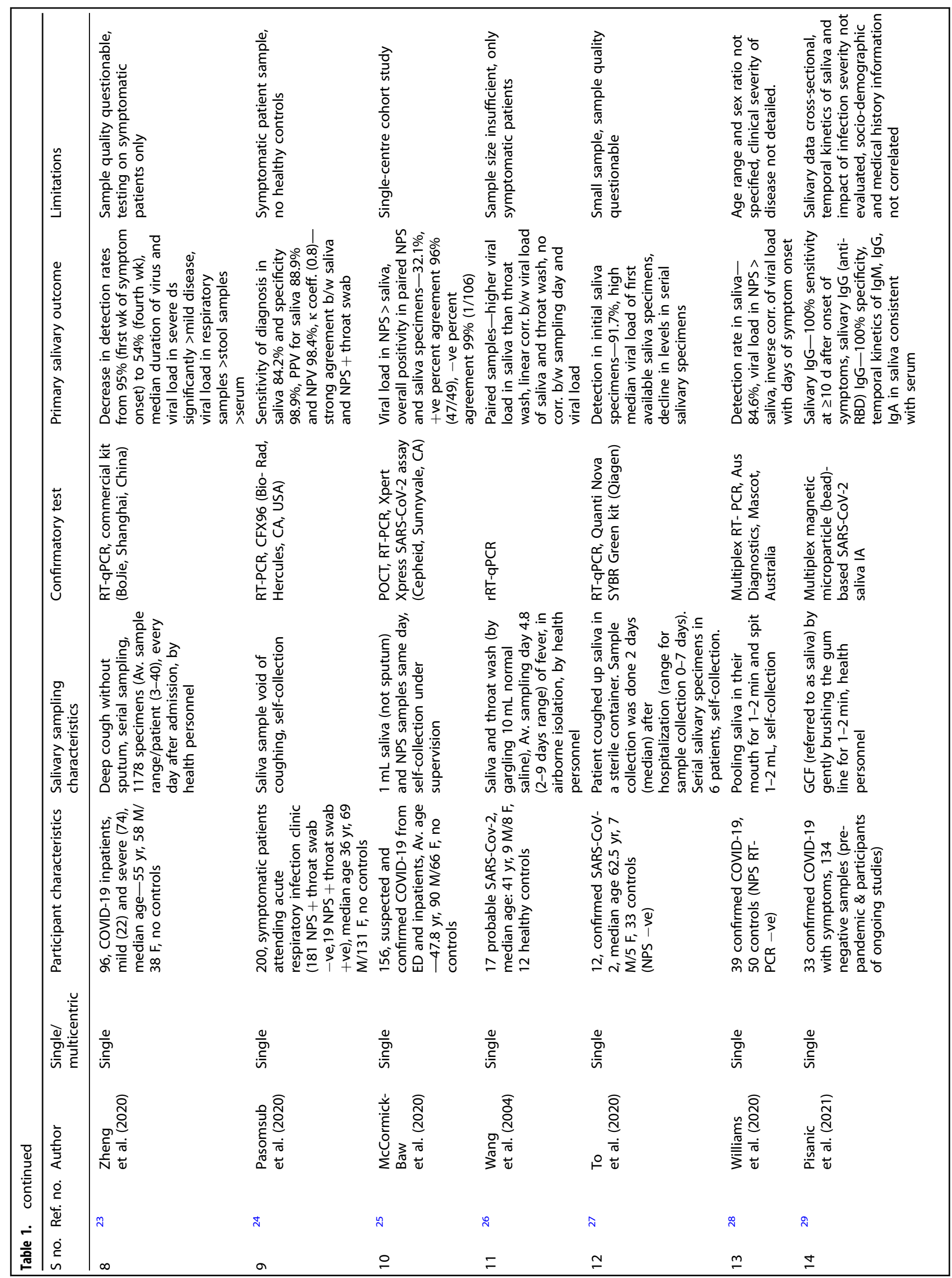




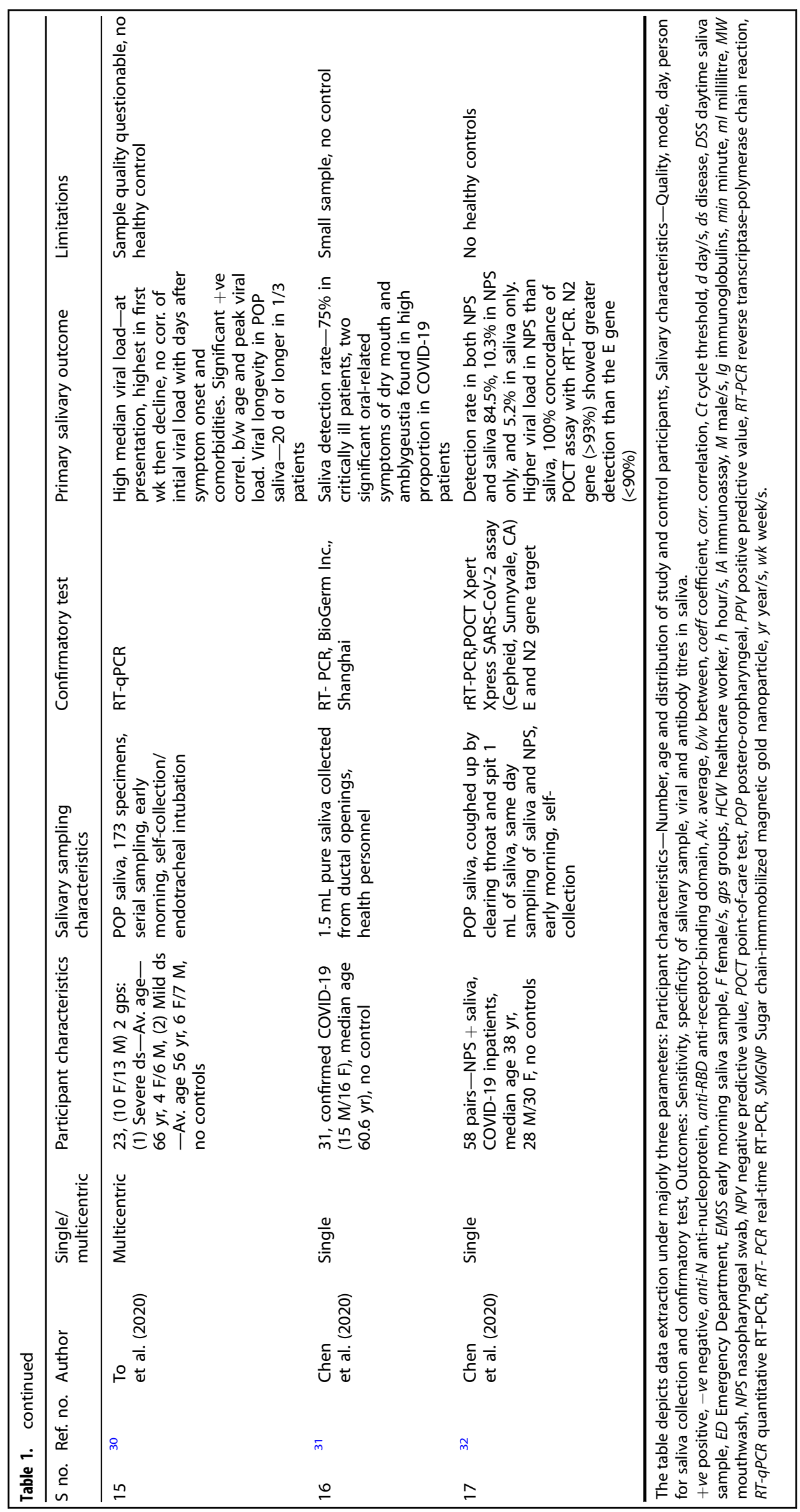


Table 2. Comparison of parameters in saliva and nasopharyngeal swabs.

\begin{tabular}{|c|c|c|}
\hline Parameters & Specific characteristics & Results \\
\hline & & Detection of ORF1ab and nCoV-N in NPS $>$ saliva $^{31}$ \\
\hline \multirow[t]{3}{*}{ Mean viral titre } & Salivary viral load $>$ NPS & $\begin{array}{l}\text { SARS-CoV-2 RNA copies in saliva (mean } \log _{10} 5.58 \text { ) gretaer than NPS } \\
\left(\text { mean } \log _{10} 4.93\right)^{16}\end{array}$ \\
\hline & Salivary viral load $<$ NPS & $\begin{array}{l}\text { Study on neonate: a steep difference in viral load level in NPS than in saliva in early } \\
\text { stages of the disease }\end{array}$ \\
\hline & & $\begin{array}{l}\text { Significantly high viral titres with significantly low Ct values in NPS than in saliva at all } \\
\text { time points }\end{array}$ \\
\hline \multirow[t]{2}{*}{ Viral sensitivity } & $\begin{array}{l}\text { Positive results in saliva and not in NPS on } \\
\text { same day collection }\end{array}$ & $\begin{array}{l}\text { Multiple studies reported the finding in paired NPS and salivary samples: }(n=1)_{1}^{25,28} \\
(n=2),{ }^{17,24}(n=3)^{32}\end{array}$ \\
\hline & & $\begin{array}{l}\text { A single case report of two male patients: }>60 \text { years, showed positive salivary sample } \\
\text { on day } 10 \text { and } 26 \text { after hospital admission, whereas } 2 \text { consecutive NPS samples came } \\
\text { negative }\end{array}$ \\
\hline POCT & Viability of saliva for POCT & $\begin{array}{l}\text { Two studies, one study showed high }+ \text { ve percent agreement b/w NPS and saliva } \\
(96 \%, 47 / 49 \text { positive samples })^{25} \text { and high }- \text { ve percent agreement }(99 \%, 1 \text { sample }+ \text { ve } \\
\text { for saliva out of } 106 \text { samples - ve for NPS), }{ }^{25} \text { the second study showed + ve testing of } \\
\text { virus in both saliva and NPS (49/58), greater in NPS only }[10.3 \%(6 / 58)] \text { compared to } \\
\text { saliva only }[5.2 \%(3 / 58)]^{32}\end{array}$ \\
\hline
\end{tabular}

The parameters of gene targets, mean viral load, and sensitivity have been compared between saliva and NPS.

$+v e$ positive, $-v e$ negative, $b / w$ between, $C l$ confidence interval, $C t$ cycle threshold, NPS nasopharyngeal swab, PCR polymerase chain reaction, POCT point-ofcare testing.

first week samples (average 4.8 days), before the development of lung lesions and slight fall by day $9 .^{26}$

Another study identified the difference in viral load between severe and mild diseases with the virus levels being higher in severe than the mild disease $(p=0.03)$ and remained high until the third and fourth weeks. ${ }^{23}$ The mild disease pattern, however, followed the previous studies with high initial viral load in the first 1 week with a peak in salivary viral load in the second week, followed by a decrease. The severe disease pattern showed high levels even in the third and fourth weeks. ${ }^{23}$

The results of serial specimen studies, in general, revealed a trend of high salivary viral load during initial 1 week of onset of symptoms, ${ }^{19,23,26,30}$ but the viral load in endotracheal aspirate of few ambulatory patients did not decrease with time, indicating a continuous high viral load in the lower respiratory tract. ${ }^{30}$ Only one 65-year-old female patient with pneumonia on lopinavir/ ritonavir $400 \mathrm{mg} / 100 \mathrm{mg}$ treatment showed fall in viral load from day 1 of hospital admission (day 4 of illness), although the levels still remained consistently high till sixth day of hospital admission. ${ }^{19}$

Studies did not support change in initial viral load in saliva with period elapsed from symptom onset. ${ }^{17,26,30}$ Contrastingly, a single cross-sectional study analysing saliva and NPS samples from the COVID screening clinic reported an inverse relation of viral load with days from symptom onset. ${ }^{28}$

\section{Salivary/serum antibody response}

The seropositivity was detected after 10 days of symptom onset with immunoglobulin $\mathrm{G}(\mathrm{IgG})$ values greater than $\operatorname{lgM}$ both for anti-nucleoprotein (NP) and anti-receptor-binding domain (RBD), which correlated with the virus neutralisation titre also used for retrospective diagnosis. ${ }^{30}$ One study also conducted serologiocal tests on specimens collected 28 days after symptom onsetand confirmed SARS-CoV-2 infection in 13 out of 17 patients. ${ }^{26}$ One study specifically studied the antibody responses in saliva $(n=33)$ and serum $(n=206)$ samples from SARS-CoV- 2 patients. The authors reported $100 \%$ sensitivity of salivary anti-NP IgG at $\geq 10$ days after onset of symptoms and $100 \%$ specificity of antiRBD IgG, with their antibody response concurrent with serum. ${ }^{29}$

Viral load sensitivity in saliva

Detection of SARS- CoV-2 virus in salivary/respiratory samples varied in studies with the duration from disease onset, quality of the sample (pure saliva or mixed with sputum/bronchopulmonary secretions), and disease severity. Studies showed high detection of the virus in saliva at $100 \%$ (25 severe disease patients, ${ }^{17} 96$ patients of severe and mild disease), ${ }^{23}$ at $91.7 \%$ in 12 patients, ${ }^{27}$ and $75 \%$ in 4 critically ill patients. ${ }^{31}$ There was a gradual decrease in sensitivity from 95 to $54 \%$ from the first week to the fourth week of symptom onset, and the decrease is significant in severe compared to mild disease. ${ }^{23}$ Studies with paired saliva and NPS samples showed a high positive percent agreement of $84.5 \%{ }^{32}$ and $96 \%{ }^{25}$ in both the samples. The overall positivity of paired samples was found to be $32.1 \%$ in probable SARS-CoV-2 patients (50 out of 156). ${ }^{25}$

Viral longevity in saliva

The longevity of the virus in salivary/respiratory sample of mild and severe disease patients have been investigated in 2 studies, which depict an average of $18-20$ days. $^{23,30}$ Of these, 1 study also 
distinguishes significantly prolonged detection of the virus in severe [21 days, interquartile range (IQR) 14-30 days] than the mild disease [14 days, IQR 10-21 days; $p=0.04]^{23}$ Another study on 2 patients documented the virus in saliva up to the 9th day of hospitalisation/13th day of illness. ${ }^{19}$ A case report in a 71-year-old patient reported virus after 37 days of symptom onset, despite the patient becoming asymptomatic. ${ }^{18}$ Similarly, 1 patient in a cohort of 12 hospitalised patients showed viral load shedding up to 11 days of hospitalisation. ${ }^{27}$ A singular study on neonate depicted undetectable viral load in saliva after 10-11 days as graphically depicted but remained detectable in NPS, stool, and urine. ${ }^{20}$

\section{Viral load quantification}

Viral load was assessed by viral count as copies $/ \mathrm{mL}$, where 4 studies mentioned high initial $\log _{10}$ values $\left(>5 \log _{10}\right), 19,26,27,30$ and $1 \log _{10}$ copies $/ \mathrm{mL}$ were labelled as undetectable. ${ }^{30}$ Other studies considered $\mathrm{Ct}$ value for viral load determination and categorised samples as negative when $\mathrm{Ct}$ values exceeded $33,{ }^{17}$ $35^{31}$ and $38 .^{16,23,24,31}$ Few studies have used $\mathrm{Ct}$ values for calculating RNA copies (copies/mL), using a dilution of plasmid DNA for generating the standard curve. ${ }^{16,19}$ Specific gene targets have been compared in two studies on saliva. Chen et al. demonstrated slightly higher detection rate (93.1\%) and $\mathrm{Ct}$ value, (IQR: 29.9-38.6) for N2 gene than E gene target (IQR 27.2-37.2), whereas McCormick-Baw et al. demonstrated average Ct values of $30.40 \pm 9.67$ for N2 gene and $26.10 \pm 11.20$ for E gene. ${ }^{25,32}$

Saliva as an alternate to standard NPS sample

Studies have been done to compare SARS-Cov- 2 detection in paired samples of saliva and NPS, the percentage of the total sample, viral load, or a combination of these parameters. ${ }^{16,17,19-}$ $22,24,25,27,28,32$ Studies majorly revealed a higher viral load in NPS than in saliva specimens with lower $\mathrm{Ct}$ values and higher $\log _{10}$ count. $19,20,24,25,32$ The comparison of parameters in saliva and NPS have been outlined in Table 2. Two studies targeted point-of-care testing (POCT) in saliva.

Associations with salivary viral load

A significant positive correlation of age with peak viral load in saliva $(p=0.02)^{30}$ as well as the duration of the infection, which was found significantly increased in severe disease patients aged $>60$ years than $<60$ years $(p=0.01) .{ }^{23}$ A single study showed no correlation of $\mathrm{Ct}$ values with age $(p=0.34) .{ }^{17}$ Contrasting results related to sex predilection were obtained where one study showed no correlation of $\mathrm{Ct}$ values with sex $(p=0.31)$ or comorbidities, ${ }^{17}$ while another reported a significantly longer duration of the infection in men than in women in severe disease patients $(p=0.01) .^{23}$

\section{Salivary sampling characteristics}

Early morning saliva before tooth brushing and breakfast has been preferred as a test sample, ${ }^{18,30,32}$ as during night in the supine position, the nasopharyngeal and bronchopulmonary secretions get collected in the posterior oropharyngeal area. The secretions can be collected by deep cough, ${ }^{23,30,32}$ spitting, ${ }^{16,18,28}$ or gargling saline. ${ }^{26}$ Two of the studies used the drooling technique to collect saliva to eliminate the oropharyngeal secretions, ${ }^{17,21}$ while one study collected salivary swabs from the opening of the salivary gland duct. $^{31}$ One study used a collective respiratory sample, including both saliva and sputum samples. ${ }^{23}$ Tajima et al. compared early morning saliva samples (EMSSs) with daytime saliva samples (DSSs) and found $66.7 \%$ sensitivity in EMSSs (4/6) compared to $25.0 \%(2 / 8)$ in DSSs, both EMSS and DSS had a similar specificity (100\%), though the sensitivity of EMSS was much better than DSS. ${ }^{18}$ Avoidance of food, drink, tobacco, or gum for $30 \mathrm{~min}$ before saliva collection has been followed in one study. ${ }^{25}$ Room conditions of airborne isolation have also been considered in one study. $^{26}$
Some clinical parameters of intervention have also been studied singularly, with 1 study testing the effect of chlorhexidine mouth wash $(0.12 \%, 15 \mathrm{~mL})$ for $30 \mathrm{~s}$ on viral load reporting a transient decrease in load in $2 \mathrm{~h}$ post gargling and then increase in $2-4 \mathrm{~h}^{19}$

\section{DISCUSSION}

For molecular diagnostics of SARS-CoV-2, NPS or throat swabs are standard samples. However, collection of the throat swabs or NPS may induce sneezing, coughing, and expelling virus particles, which exacerbate health hazards for HCWs. Moreover, the collection of these swabs is a relatively invasive procedure that causes significant patient discomfort and may also induce bleeding in tonsils and posterior pharynx. NPS collection procedure is time-consuming, requires consumables, specialised set-up, and can be done by trained professionals only. Further, as the pandemic magnifies in intensity, the requirement of mass screening in densely populated locations, more so in poor and developing countries, is rising. Huge global demand for swabs is expected in the near future. With the above constraints of NPS, an alternate easy-to-use, less technique-sensitive, but reliable diagnostic sample is the need of the day.

Herein, based on a careful appraisal and interpretation of literature, we support the hypothesis that saliva can be a viable sample for the molecular diagnosis of SARS-CoV-2. Sufficient evidence has been generated in the present scoping review related to salivary specimens in SARS-Cov-2 for viral replication, longevity, sensitivity, specificity with other related viruses, and its practicality in the collection of specimens.

Tracking viral load can be vital in monitoring SARS-CoV-2 infections, risk assessment for infectivity, morbidity, clinical prognosis, and mortality. The results of the current scoping review showed a trend of high viral load in the first week of symptom onset with an approximate count of $5.2 \log _{10}$ copies $/ \mathrm{mL}$ and a subsequent decline in the levels thereafter. ${ }^{30}$ Concurrently, Wolfel et al. reported high pharyngeal virus shedding with average value of $6.76 \times 10^{5}$ copies/whole swab $\left(>5.5 \log _{10}\right)$ in NPS and throat swab samples of COVID-19-positive patients during the first week of symptoms, with a peak on fourth day and a fall in viral load after day $5 .{ }^{33}$ This pattern of rise and subsequent decline of viral burden in throat swabs was also found in a single study included in the current review, which showed full concordance in paired salivary and throat swab samples. ${ }^{26}$

Ct value in the RT-PCR test has for long been considered to be relatively accurate for viral load determination. One of the studies of the current review showed a mean $\mathrm{Ct}$ value of $27.16+/-3.07$, in the initial one week of symptom onset. ${ }^{17}$ This was concordant with aggregated $\mathrm{Ct}$ values in NPS by Zou et al., where lower $\mathrm{Ct}$ values/higher viral load were found in the initial 1 week of symptom onset. ${ }^{34}$ The $\mathrm{Ct}$ value was also found in positive correlation with days from symptom onset in a single crosssectional study collecting saliva and NPS samples from the COVID19 screening clinic. ${ }^{28}$ A similar decrease was noted in the viral load of sputum and swab (NPS and throat swab) samples. ${ }^{33,34}$

Detection of active virus in salivary/respiratory sample have been reported in studies for average 18-20 days in mild and severe disease patients. ${ }^{23,30}$ Wolfel et al. ${ }^{33}$ reported the isolation of the live virus in the first week of symptoms in a high percentage of samples [swabs (16.66\%), sputum (83.33\%)] and not isolated after day 8, although viral load was high. This study showed longevity of upper respiratory swab samples until 28 days, ${ }^{33}$ similar to a single case report of a 71-year-old male patient where the virus was detected until 37 days in saliva. ${ }^{18}$ The higher viral load and duration of the SARS CoV-2 virus in older age is suggested as being related to immunosenescence and a greater level of ACE2, a potent novel CoV receptor. ${ }^{35,36}$ The salivary viral load can be further influenced by the level of ACE2 receptors in various sites of the oral mucosa, epithelial cells of salivary glands, and lung alveoli. ${ }^{17,23,24}$ 
On correlating disease severity with viral load, studies on salivary samples have indicated higher viral load in severe than in mild disease. ${ }^{23,30}$ A study on upper respiratory tract samples also supported these findings with mean $\mathrm{Ct}$ values lower in severe disease patients than mild-moderate disease by 2.8 (95\% confidence interval $(\mathrm{Cl}),-2.4$ to 8.0$)$ and $2.5(95 \% \mathrm{Cl},-0.8$ to 5.7$)$ in nasal and throat swabs, respectively. ${ }^{34}$

Sex predilection proved greater disease severity and prolonged duration of the virus in males compared to females, ${ }^{23}$ as was reported in infection with other pathogenic CoVs (SARS and MERS). ${ }^{37}$ This difference was attributed to lower immune status in men than in women owing to the difference in hormone levels. ${ }^{38}$

Gargling with chlorhexidine compounds $(0.12 \%, 15 \mathrm{~mL})$ for suppression of lipid-enveloped CoVs has been proposed in one of the studies, where a transient decrease in salivary viral load was reported for $2 \mathrm{~h}$, which regained $2-4 \mathrm{~h}$ post-mouthwash. ${ }^{19}$ Although this was a single study on two hospitalised patients, the effect of mouthwashes on SARS-CoV-2 deserves a separate study and review as work has been progressing on mouthwash use in COVID-19, both in hospital and community set-up.

Possibility of self-collection of the saliva specimen is a major advantage over NPS, thus reducing the risk of contamination to HCWs and being non-invasive and comfortable for the patient. $^{16,18,19,24,25,27-30,32}$ Time and cost reduction in saliva compared to NPS sampling has been reported as 1.38- and 2.09fold, respectively, with mean saliva sample collection time of $114 \mathrm{~s}$ and mean cost for a single sample of $\$ 1.16 .^{39} \mathrm{~A}$ comparison of NPS and saliva has been made in multiple studies in the current scoping review, where saliva has proved equally effective as a diagnostic specimen. ${ }^{16,17,19,32}$ Few studies showed positive salivary samples even after NPS was negative, $16,17,21,24$ and a single study reported higher levels of viral titres in saliva than in NPS. ${ }^{16}$ One study has proposed that two negative NPS should be supplemented with an additional negative salivary sample at the time of hospital discharge. ${ }^{17}$

A very recent study by Wyllie et al. ${ }^{16}$ found a higher percentage of viral positivity in saliva compared to NPS in 1-5 days and 6-10 days from COVID-19 diagnosis in 70 hospitalized pateints, whereas samples collected after 11 days found the percentages reversed. The viral titres were also significantly higher in saliva than in NPS in the first available samples. ${ }^{16}$

POCT, used in two studies in the current review, is known to assist in rapid clinical decisions and decrease variations in specimen handling. POCT by the Xpert assay in one study showed $84.5 \%(49 / 58)$ positive paired samples of saliva and NPS ${ }^{32}$ and another study a positive percent agreement of $96 \%$ (86.02-99.5\%, $95 \% \mathrm{Cl}$ ) of saliva and $\mathrm{NPS}^{25}$ with no statistically significant difference. Although the viral load in saliva is less compared to NPS, ${ }^{19,20,24,25,27,28,32}$ a few paired sample studies showed detection in saliva and not in NPS; therefore, saliva is suggested to be used as a viable first-line screening test in low resources, multiple environments, and ambulatory at-risk patients. ${ }^{28}$

Salivary antibody surveillance as a non-invasive alternate for serological testing has shown some evidence in the current scoping review. Salivary antibodies are shown to rise $\geq 10$ days after symptom onset, with a high sensitivity and specificity of salivary lgG (anti-RBD). ${ }^{29,30}$ Consistency in temporal kinetics of Igs in serum and saliva (IgM, $\operatorname{lgG}, \lg \mathrm{A}$ ) was also observed. ${ }^{29}$ Hence, salivary antibody profiling can be proposed for widespread community-based monitoring of SARS-CoV-2.

Meanwhile, newer second-generation RT-PCR kits are being used to minimise the false-negative results in saliva and increase the SARS-CoV2 detection sensitivity, in the range of $67.1-97 \%$ and specificity of $100 \% .{ }^{40-42}$ Although these studies were beyond the scope of this review, these kits can offer enhanced diagnostic sensitivity in salivary samples, and future studies should focus on these kits.
The conditions for saliva collection-time, temperature, humidity-have not been given due importance in the studies. One study mentioned higher viral load and higher sensitivity and specificity of SARS-CoV-2 virus in EMSS compared to DSS, ${ }^{18}$ which can be attributed to decreased viral clearance due to cessation of salivary outflow during sleep in early morning samples. ${ }^{43}$ Additionally, DSS can show increased salivary volume and decrease in inhibitory agents to RT-PCR like food, haemoglobin, and various body fluids. ${ }^{44}$

One study reported the rapid spread of infection in local family clusters with the percentage of confirmed cases increasing from $7.7 \%$ between days 22 and 32 to $93.1 \%$ between days 33 and 42 . In addition, authors suggested the importance of prevention of nosocomial infection mentioning strict criteria of isolation to prevent transmission in airborne infection isolation rooms or a minimum $1 \mathrm{~m}$ distance between patients for contact, droplet, and airborne precautions. Practices like the discussion of concerns and education of staff in open forums or update of infection control measures were also mentioned. ${ }^{22}$

This scoping review also brings forth some new interesting findings about oral symptoms, which may be of particular interest to dentists and also could prove to be initial warning signs of COVID-19. These oral symptoms like dry mouth, amblygeustia, dryness, and inflammation of the mouth and submandibular lymph nodes enlargement can be attributed to high ACE2 in tongue epithelial cells, providing a possible entry route to SARSCoV-2, besides being present in salivary glands. ${ }^{31}$ Thus oral symptoms and hygiene can attain a crucial role in diagnosing and preventing COVID-19 and requires further exploration.

Limitations of the study

Although the present review highlights the available evidence in the viability of saliva as a clinical specimen for diagnosis of SARSCov-2, the studies are not without limitations. This review included case reports in the review ${ }^{18-21}$ as they provide excellent insights into some new domains of COVID-19 diagnostics and treatment aspects, like the use of chlorhexidine mouthwash protocol, EMSS better than daytime saliva, detection of virus till 37 days in an old male patient with allergic rhinitis, and viral titres in neonate higher than in mother and persisting in stool longer than respiratory samples.

Keeping in view the current pandemic crisis, the initial diagnostic criteria and patient classification were understandably heterogeneous and non-standardised. There is not enough evidence whether saliva in asymptomatic infected people would carry viral RNA to enable molecular detection as only one study had a control group of asymptomatic HCWs. The majority of the studies were single-centre COVID-19 cohorts, which presented a risk of bias and could overinflate estimates of viral load. Serial salivary samples for temporal evaluation were present in a few studies, but there was a lack of uniformity in the day of salivary sample collection. Hence, the influence of days of symptom onset on salivary viral load remains unresolved. This scoping review also highlights gaps in protocols for saliva sample collection.

\section{CONCLUSIONS}

Critical appraisal of literature has brought forth the following inferences:

1. Studies evaluating viral load in paired NPS/saliva samples or serial saliva samples have documented high SARS-CoV-2 viral load in saliva ( $>5 \log 10$ average) in the first week of symptom onset.

2. Studies evaluating SARS-CoV-2 in saliva with grades of disease severity have shown a significantly increased median duration of virus (2-3 weeks) and higher viral load in patients of severe disease, with or without comparison to mild disease. 
3. Studies evaluating N2 and E gene targets in saliva depicted higher detection rate and lower viral load (greater $\mathrm{Ct}$ value) in the former and, between ORF1ab and nCoV-N gene, greater detection of $\mathrm{N}$ gene.

4. A positive correlation of peak viral $\log 10$ value was found with age, more so above 60 years. Sex predilection for the duration of the virus in saliva favoured males more than females, although some studies have negated any correlation with age, sex, or comorbidities.

5. While majority of studies evaluating viral load in paired saliva/ NPS samples found a greater sensitivity in NPS than in saliva, few studies reported positive results in saliva, not in NPS, on the same day, which needs further exploration and verification.

6. Studies evaluating the accuracy of POCT in saliva showed high positive percent agreement between PCR-positive NPS and saliva samples.

The findings of this scoping review suggest that saliva can be implored as a viable adjunct specimen in the initial screening of SARS CoV-2 in community or hospital set-up.

\section{Recommendations}

We recommend that future studies on saliva should consider a robust research protocol keeping in view the limitations of earlier studies.

1. Saliva collection protocol for quality assurance should include a serial sampling of saliva, and preferably early morning saliva, before brushing and eating. The saliva should be collected with passive drool, void of coughing. The saliva collection can be either clinician supervised self-collection or with recorded (video/audio) instructions and remote monitoring.

2. Studies should consider a control asymptomatic or healthy group matched for age and sex.

Further studies should as well address the gaps in existing knowledge, notably viral load in the saliva of asymptomatic patients, viral load in correlation with comorbidities and treatments, oral symptoms, age, and sex considerations. Investigation on the presence of salivary COVID-19 viral load in children is also warranted.

\section{ACKNOWLEDGEMENTS}

The research in the Laboratory of Infection Biology and Translational Research is supported by the AlIMS Intramural grant (A-638) and HarGobind Khorana Innovative Young Biotechnologist Award (BT/11/IYBA/2018/01) [both to V.S.].

\section{AUTHOR CONTRIBUTIONS}

P.K., A.C., D.B.P.: contributed to conception, design, data acquisition, analysis and interpretation, drafted and critically revised the manuscript. O.P.K.: contributed to conception, design, analysis and interpretation, reviewed drafts, and critically revised the manuscript. K.G., V.S.: contributed to analysis and interpretation, drafted and critically revised the manuscript. All authors gave their final approval and agreed to be accountable for all aspects of the work.

\section{ADDITIONAL INFORMATION}

Competing interests: The authors declare no competing interests.

Publisher's note Springer Nature remains neutral with regard to jurisdictional claims in published maps and institutional affiliations.

\section{REFERENCES}

1. Lu, R. et al. Genomic characterisation and epidemiology of 2019 novel coronavirus: implications for virus origins and receptor binding. Lancet 395, 565-574 (2020).
2. Naji, H. S. The emerging of the 2019 novel coronavirus 2019-nCoV. Eur. J. Med. Health Sci. https://doi.org/10.24018/ejmed.2020.2.1.169 (2020).

3. Chen, N. et al. Epidemiological and clinical characteristics of 99 cases of 2019 novel coronavirus pneumonia in Wuhan, China: a descriptive study. Lancet 395, 507-513 (2020).

4. Fan, Y. et al. Bat coronaviruses in China. Viruses 11, 27-32 (2019)

5. Fehr, A. R. \& Perlman, S. Coronaviruses: an overview of their replication and pathogenesis. Methods Mol. Biol. 1282, 1-23 (2015).

6. Chowdhry, A., Kapoor, P. \& Popli, D. B. Strengthening health care research and academics during and after COVID19 pandemic- an Indian perspective. J. Oral. Biol. Craniofacial Res. 10, 343-346 (2020).

7. Cucinotta, D. \& Vanelli, M. WHO declares COVID-19 a pandemic. Acta Biomed. 91, 157-160 (2020)

8. Wrapp, D. et al. Cryo-EM structure of the 2019-nCoV spike in the prefusion conformation. Science 367, 1260-1263 (2020).

9. Wan, Y. et al. Receptor recognition by the novel coronavirus from Wuhan: an analysis based on decade-long structural studies of SARS coronavirus. J. Virol. 94, e00127-20 (2020).

10. Long, C. et al. Diagnosis of the coronavirus disease (COVID-19): rRT-PCR or CT? Eur. J. Radiol. 126, 108961 (2020).

11. Pang, J. et al. Potential rapid diagnostics, vaccine and therapeutics for 2019 novel coronavirus (2019-nCoV): a systematic review. J. Clin. Med. 9, 623-656 (2020).

12. Qian, Y. et al. Safety management of nasopharyngeal specimen collection from suspected cases of coronavirus disease 2019. Int. J. Nurs. Sci. 7, 153-156 (2020).

13. Kim, Y.-G. et al. Comparison between saliva and nasopharyngeal swab specimens for detection of respiratory viruses by multiplex reverse transcription-PCR. J. Clin. Microbiol. 55, 226-233 (2017).

14. Sabino-Silva, R., Jardim, A. C. G. \& Siqueira, W. L. Coronavirus COVID-19 impacts to dentistry and potential salivary diagnosis. Clin. Oral. Investig. 24, 1619-1621 (2020).

15. Vinayachandran, D. \& Saravanakarthikeyan, B. Salivary diagnostics in COVID-19: future research implications. J. Dent. Sci. 15, 364-366 (2020).

16. Wyllie, A. L. et al. Saliva or Nasopharyngeal swab specimens for detection of SARS-CoV-2. N Engl J Med. 383, 1283-1286 (2020).

17. Azzi, L. et al. Saliva is a reliable tool to detect SARS-CoV-2. J. Infect. 81, e45-e50 (2020).

18. Tajima, Y., Suda, Y. \& Yano, K. A case report of SARS-CoV-2 confirmed in saliva specimens up to 37 days after onset: proposal of saliva specimens for COVID-19 diagnosis and virus monitoring. J. Infect. Chemother. 26, 1086-1089 (2020).

19. Yoon, J. G. et al. Clinical significance of a high SARS-CoV-2 viral load in the saliva. J. Korean Med. Sci. 35, e195 (2020).

20. Han, M. S. et al. Sequential analysis of viral load in a neonate and her mother infected with severe acute respiratory syndrome coronavirus 2. Clin. Infect. Dis. 71, 2236-2239 (2020).

21. Azzi, L. et al. Two cases of COVID-19 with positive salivary and negative pharyngeal or respiratory swabs at hospital discharge: a rising concern. Oral Dis. https://doi.org/10.1111/odi.13368 (2020).

22. Cheng, V. C. C. et al. Escalating infection control response to the rapidly evolving epidemiology of the coronavirus disease 2019 (COVID-19) due to SARS-CoV-2 in Hong Kong. Infect. Control Hosp. Epidemiol. 41, 493-498 (2020).

23. Zheng, S. et al. Viral load dynamics and disease severity in patients infected with SARS-CoV-2 in Zhejiang province, China, January-March 2020: retrospective cohort study. BMJ 369, m1443 (2020).

24. Pasomsub, E. et al. Saliva sample as a non-invasive specimen for the diagnosis of coronavirus disease 2019: a cross-sectional study. Clin. Microbiol. Infect. https://doi.org/10.1016/j.cmi.2020.05.001 (2020).

25. McCormick-Baw, C. et al. Saliva as an alternate specimen source for detection of SARS-CoV-2 in symptomatic patients using Cepheid Xpert Xpress SARS-CoV-2. J. Clin. Microbiol. https://doi.org/10.1128/JCM.01109-20 (2020).

26. Wang, W.-K. et al. Detection of SARS-associated coronavirus in throat wash and saliva in early diagnosis. Emerg. Infect. Dis. 10, 1213-1219 (2004).

27. To, K. K. et al. Consistent detection of 2019 novel coronavirus in saliva. Clin. Infect. Dis. 71, 841-843 (2020)

28. Williams, E. et al. Saliva as a non-invasive specimen for detection of SARS-CoV-2. J. Clin. Microbiol. 58, e00776-20 (2020).

29. Pisanic, N. et al. COVID-19 serology at population scale: SARS-CoV-2-Specific antibody responses in saliva. J Clin Microbiol. 59, e02204-e02220 (2020).

30. To, K. K.-W. et al. Temporal profiles of viral load in posterior oropharyngeal saliva samples and serum antibody responses during infection by SARS-CoV-2: an observational cohort study. Lancet Infect. Dis. 20, 565-574 (2020).

31. Chen, L. et al. Detection of SARS-CoV-2 in saliva and characterization of oral symptoms in COVID-19 patients. Cell Prolif. 53, e12923 (2020).

32. Chen, J. H.-K. et al. Evaluating the use of posterior oropharyngeal saliva in a point-of-care assay for the detection of SARS-CoV-2. Emerg. Microbes Infect. 9 , 1356-1359 (2020). 
33. Wölfel, R. et al. Virological assessment of hospitalised patients with COVID-2019. Nature 581, 465-469 (2020).

34. Zou, L. et al. SARS-CoV-2 viral load in upper respiratory specimens of infected patients. N. Engl. J. Med. 382, 1177-1179 (2020).

35. Li, G. et al. Assessing ACE2 expression patterns in lung tissues in the pathogenesis of COVID-19. J. Autoimmun. 112, 102463 (2020).

36. Pera, A. et al. Immunosenescence: implications for response to infection and vaccination in older people. Maturitas 82, 50-55 (2015).

37. Alghamdi, I. G. et al. The pattern of Middle East respiratory syndrome coronavirus in Saudi Arabia: a descriptive epidemiological analysis of data from the Saudi Ministry of Health. Int. J. Gen. Med. 7, 417-423 (2014).

38. Channappanavar, R. et al. Sex-based differences in susceptibility to severe acute respiratory syndrome coronavirus infection. J. Immunol. 198, 4046-4053 (2017).

39. To, K. K. W. et al. Saliva as a diagnostic specimen for testing respiratory virus by a point-of-care molecular assay: a diagnostic validity study. Clin. Microbiol. Infect. 25, 372-378 (2019).

40. Drosten, $C$. et al. Evaluation of advanced reverse transcription-PCR assays and an alternative PCR target region for detection of severe acute respiratory syndromeassociated coronavirus. J. Clin. Microbiol. 42, 2043-2047 (2004).

41. Yip, C. C.-Y. et al. Evaluation of the commercially available LightMix ${ }^{\circledR}$ Modular Egene kit using clinical and proficiency testing specimens for SARS-CoV-2 detection. J. Clin. Virol. 129, 104476 (2020).
42. Wei, S. et al. Field-deployable, rapid diagnostic testing of saliva samples for SARSCoV-2. Preprint at medRxiv https://doi.org/10.1101/2020.06.13.20129841 (2020).

43. Humphrey, S. P. \& Williamson, R. T. A review of saliva: normal composition, flow, and function. J. Prosthet. Dent. 85, 162-169 (2001).

44. Schrader, C. et al. PCR inhibitors-occurrence, properties and removal. J. Appl. Microbiol. 113, 1014-1026 (2012).

(C) Open Access This article is licensed under a Creative Commons Attribution 4.0 International License, which permits use, sharing, adaptation, distribution and reproduction in any medium or format, as long as you give appropriate credit to the original author(s) and the source, provide a link to the Creative Commons license, and indicate if changes were made. The images or other third party material in this article are included in the article's Creative Commons license, unless indicated otherwise in a credit line to the material. If material is not included in the article's Creative Commons license and your intended use is not permitted by statutory regulation or exceeds the permitted use, you will need to obtain permission directly from the copyright holder. To view a copy of this license, visit http://creativecommons. org/licenses/by/4.0/.

(c) The Author(s) 2021 